\title{
Magnetic susceptibility and magnetization properties of asymmetric nuclear matter in a strong magnetic field
}

\author{
A. Rabhi, ${ }^{1,2,{ }^{*}}$ M. A. Pérez-García, ${ }^{3, \dagger}$ C. Providência,,${ }^{1, \ddagger}$ and I. Vidaña ${ }^{1, \S}$ \\ ${ }^{1}$ Centro de Física Computacional, Department of Physics, University of Coimbra, 3004-516 Coimbra, Portugal \\ ${ }^{2}$ University of Tunis El-Manar, Unité de Recherche de Physique Nucléaire et des Hautes Énergies, \\ Faculté des Sciences de Tunis, 2092 Tunis, Tunisia \\ ${ }^{3}$ Departamento de Física Fundamental and IUFFyM, Universidad de Salamanca, Salamanca, Spain \\ (Received 10 October 2014; revised manuscript received 25 February 2015; published 17 April 2015)
}

\begin{abstract}
We study the effects of a strong magnetic field on the proton and neutron spin polarization and magnetic susceptibility of asymmetric nuclear matter within a relativistic mean-field approach. It is shown that magnetic fields $B \sim 10^{16}-10^{17} \mathrm{G}$ have noticeable effects on the range of densities of interest for the study of the crust of a neutron star. Although the proton susceptibility is larger for weaker fields, the neutron susceptibility becomes of the same order or even larger for small proton fractions and subsaturation densities for $B>10^{16} \mathrm{G}$. We expect that neutron superfluidity in the crust will be affected by the presence of magnetic fields.
\end{abstract}

DOI: 10.1103/PhysRevC.91.045803

PACS number(s): 97.60.Jd, 26.60.-c, 24.10.Jv, 21.65.-f

\section{INTRODUCTION}

A well-grounded understanding of the properties of isospinasymmetric nuclear systems, such as nuclei far from the stability valley or astronomical objects like neutron stars, is crucial for the advancement of both nuclear physics and astrophysics [1-4]. A major experimental and theoretical effort with significant progress has been carried out in recent years (see, e.g., Ref. [1] and references therein) on the study of the properties of isospin-asymmetric nuclear systems. Laboratory experiments, such as those recently performed or being planned in existing or next-generation radioactive ion beam facilities, are providing crucial information on the isospin dependence of the nuclear force from a large number of heavy ion collision and nuclear structure observables. Complementary information can be extracted from the observation of neutron stars, which open a window into both the bulk and microscopic properties of nuclear matter at extreme isospin asymmetries.

Neutron stars, most of which are detected as pulsars, have strong surface magnetic fields which can reach values of the order of $10^{14}-10^{15} \mathrm{G}$ in the case of the so-called magnetars ${ }^{1}$ that may grow by several orders of magnitude in the dense interior of the star. Despite the great theoretical effort of the past forty years, there is still no general consensus regarding the mechanism that generates such strong magnetic fields in a neutron star. The field could be a fossil remnant from that of the progenitor star [6], or alternatively, it could be generated after the formation of the neutron star by some long-lived electric currents flowing in the highly conductive neutron star material [7]. From the nuclear physics point of view, however, one of the most interesting and stimulating mechanisms that has been suggested is the possible existence

\footnotetext{
*rabhi@teor.fis.uc.pt

†mperezga@usal.es

${ }^{\ddagger} \mathrm{cp} @$ teor.fis.uc.pt

§ividana@fis.uc.pt

${ }^{1}$ For a review of magnetars, see, e.g., Ref. [5].
}

of a phase transition to a ferromagnetic state at densities corresponding to the theoretically stable neutron stars and, therefore, of a ferromagnetic core in the liquid interior of such compact objects. Such a possibility has long been considered by many authors within different theoretical approaches (see, e.g., [8-32]), but results were contradictory. Therefore, a complete understanding of the magnetic properties of neutron stars and, more generally, of those of isospin-asymmetric nuclear matter, requires the study of nuclear matter under the influence of magnetic fields.

An estimation of the maximum magnetic field intensity supported by a star before magnetic field stresses give rise to the formation of a black hole may be obtained equating the magnetic field energy of an uniform field in a sphere with the star radius $R$ to the gravitational binding energy. Using the Schwarzschild criterion that $R>2 M \mathrm{G} / \mathrm{c}^{2}$, the magnetic field should satisfy $B \leqslant 8 \times 10^{18}\left(1.4 M_{\odot} / M\right) \mathrm{G}$ [33]. This estimation is just slightly larger than the maximum fields obtained in the framework of a relativistic magneto-hydrostatic formalism [33], which were of the order of $\sim 5 \times 10^{18} \mathrm{G}$. In Ref. [34], using the same approach and an hyperonic equation of state (EOS), the authors have obtained stable configurations for $B \leqslant 3 \times 10^{18} \mathrm{G}$. However, they did not exclude larger fields, and, in particular, they suggest that a nonconstant current function with an appreciable gradient or a disordered field with $\left\langle B^{2}\right\rangle>\langle\vec{B}\rangle^{2}$ could possibly give rise to larger fields in still stable stars. Taking these numbers as indicative, we consider fields $B \leqslant 10^{19} \mathrm{G}$ in the present work.

Until recently fields no higher than $\sim 10^{15} \mathrm{G}$ have been measured at the surface of magnetars; see Ref. [35]. ${ }^{2}$ From the observation of the spin down of pulsars via electromagnetic radiation, the surface poloidal magnetic field can be estimated. However, there is some evidence that the internal magnetic field of several external low-field magnetars could be higher. An example is SGR 0418+5729, whose dipole field is less than a few times $10^{12} \mathrm{G}$ : An internal toroidal field as large

\footnotetext{
${ }^{2}$ http://www.physics.mcgill.ca/ $/$ pulsar/magnetar/main.html
} 
as $\sim 10^{16} \mathrm{G}$ could have existed inside its interior, which would explain some of the presently observed properties [36]. Using a pure toroidal magnetic field, equilibrium models of relativistic stars have been calculated for both nonrotating and rotating stars. These toroidal fields vanish at the symmetry axis, have a maximum value that can be larger than $10^{18} \mathrm{G}$ depending on the EOS on the equatorial plane deep inside the star, and decrease toward the surface where they vanish [37]. Let us also point out that the internal poloidal fields calculated using a general relativistic magnetohydrostatic formalism are larger than the surface ones [38]. It has also been suggested that magnetic fields may be held in the core for periods much longer than the ohmic diffusion time due to interactions between the magnetic flux tubes and the vortex tubes expected to be present in a superconducting, superfluid, rotating neutron star [39]. The detailed underlying mechanism for this is, however, far from being completely understood.

Particularly interesting is the study of the magnetization of matter due to the presence of a magnetic field. Whereas the magnetization of symmetric nuclear matter and pure neutron matter has been studied by several authors [40-45], the magnetization of $\beta$-stable neutron star matter has received less attention in the literature. In Ref. [46], for instance, the magnetization of $\beta$-stable matter was extensively studied for a single-component electron gas and for the crust matter of neutron star. This study was generalized by Broderick et al. [47] by including also the contribution of neutrons and protons. Recently, Dong et al. [48] have studied the effect of density dependence of the nuclear symmetry energy on the magnetization of $\beta$-stable matter. These authors concluded that the magnetic susceptibility of charged particles (protons, electrons, and muons) can be larger than that of the neutron and that the anomalous magnetic moment of the protons enhances their magnetic susceptibility to the point that it can be one of the main contributions and therefore should not be neglected. They also show that the proton magnetic susceptibility is sensitive to the density dependence of the nuclear symmetry energy, namely to the isospin content of the nuclear force.

In this work we study the magnetization of spin-polarized isospin asymmetric nuclear matter at zero temperature by using a relativistic mean-field approach. The scope of this work is threefold: (i) to determine under which conditions of density and isospin asymmetry, matter in the presence of a magnetic field is totally polarized, (ii) to compare under such conditions the proton and neutron magnetic susceptibilities, and finally (iii) to determine which is in each case the most energetically favorable spin configuration. The density dependence of the energy of the system and its pressure, as well as its compressibility, is analyzed for different proton fractions and magnetic fields. We will not consider $\beta$-equilibrium matter in most of the results shown, but will consider a wide range of nuclear matter asymmetries of interest for stellar matter, in particular, to the study of the inner crust, where a pasta phase is expected [49], to the study of matter with trapped neutrinos where large proton fractions are expected, which may be as large as 0.4 in the presence of a magnetic field [50], and to the study of neutrino free matter in $\beta$-equilibrium where the proton fraction will increase above 0.1 at subsaturation densities for a sufficiently strong magnetic field [51]. Therefore, besides symmetric nuclear matter and neutron matter, we choose two representative proton fractions, namely $Y_{p}=0.1$ for cold $\beta$-equilibrium matter and $Y_{p}=0.3$ for warm protoneutron star matter with a fraction of 0.4 trapped leptons. For reference, we also present the proton fraction of $\beta$-equilibrium matter for the magnetic field intensities considered in the present work, as well as the proton and neutron polarization and magnetization of $\beta$-equilibrium matter for some of the cases discussed. Let us point out that even though a protoneutron star should be described at finite temperature, which has as an immediate consequence the washout of Landau levels, the main features defined by a large isospin symmetry due to neutrino trapping may be understood at zero temperature. In addition, the role of the proton anomalous magnetic moment is investigated in detail.

The paper is organized in the following way. A short review of the formalism is presented in Sec. II. In Sec. III we present explicit expressions for the magnetization of each nucleonic species, as well as for their differential susceptibilities. The results are shown and discussed in Sec. IV. Finally, a short summary and our main conclusions are given in Sec. V.

\section{THE FORMALISM}

To describe nuclear matter in a external uniform magnetic field $B$ along the $z$ axis, we employ a relativistic meanfield (RMF) approach, in which the nucleons interact via the exchange of $\sigma, \omega$, and $\rho$ mesons. The total interacting Lagrangian density of the nonlinear Walecka model (NLWM) has the form

$$
\mathcal{L}=\sum_{N=n, p} \mathcal{L}_{N}+\mathcal{L}_{m}
$$

The nucleon ( $N=n, p$ ) Lagrangian density, including meson-nucleon interacting terms, and the meson $(\sigma, \omega$, and $\rho$ ) Lagrangian density are, respectively, given by

$$
\begin{aligned}
\mathcal{L}_{N}= & \bar{\Psi}_{N}\left(i \gamma_{\mu} \partial^{\mu}-q_{N} \gamma_{\mu} A^{\mu}-m+g_{\sigma} \sigma-g_{\omega} \gamma_{\mu} \omega^{\mu}\right. \\
& \left.-\frac{1}{2} g_{\rho} \boldsymbol{\tau} \cdot \gamma_{\mu} \rho^{\mu}-\frac{1}{2} \mu_{N} \kappa_{N} \sigma_{\mu \nu} F^{\mu \nu}\right) \Psi_{N}
\end{aligned}
$$

and

$$
\begin{aligned}
\mathcal{L}_{m}= & \frac{1}{2} \partial_{\mu} \sigma \partial^{\mu} \sigma-\frac{1}{2} m_{\sigma}^{2} \sigma^{2}-\frac{1}{3 !} c_{\sigma} \sigma^{3}-\frac{1}{4 !} \lambda \sigma^{4} \\
& +\frac{1}{2} m_{\omega}^{2} \omega_{\mu} \omega^{\mu}+\frac{1}{4 !} \xi g_{\omega}^{4}\left(\omega_{\mu} \omega^{\mu}\right)^{2}-\frac{1}{4} \Omega^{\mu \nu} \Omega_{\mu \nu} \\
& -\frac{1}{4} F^{\mu \nu} F_{\mu \nu}+\frac{1}{2} m_{\rho}^{2} \rho_{\mu} \cdot \rho^{\mu}-\frac{1}{4} P^{\mu \nu} P_{\mu \nu} \\
& +\Lambda_{\omega} g_{\rho}^{2} \rho_{\mu} \cdot \rho^{\mu} g_{\omega}^{2} \omega_{\mu} \omega^{\mu} .
\end{aligned}
$$

In the above expressions, $\Psi_{N}$ are the nucleon Dirac fields, the nucleon mass is denoted by $m, \tau$ are the isospin Pauli matrices, and $\mu_{N}$ is the nuclear magneton. The nucleon anomalous magnetic moments (AMM) are introduced via the coupling of the baryons to the electromagnetic field tensor with $\sigma_{\mu \nu}=\frac{i}{2}\left[\gamma_{\mu}, \gamma_{\nu}\right]$ and strength $\kappa_{N}$, with $\kappa_{n}=-1.91315$ for the neutron and $\kappa_{p}=1.79285$ for the proton, respectively. The mesonic and electromagnetic field strength tensors are given by their usual expressions: $\Omega_{\mu \nu}=\partial_{\mu} \omega_{\nu}-\partial_{\nu} \omega_{\mu}, P_{\mu \nu}=$ 
$\partial_{\mu} \boldsymbol{\rho}_{\nu}-\partial_{\nu} \boldsymbol{\rho}_{\mu}-g_{\rho}\left(\boldsymbol{\rho}_{\mu} \times \boldsymbol{\rho}_{\nu}\right)$, and $F_{\mu \nu}=\partial_{\mu} A_{\nu}-\partial_{\nu} A_{\mu}$. The photon field $A^{\mu}$ is taken as $(0,0, B x, 0)$ in such a way that the external magnetic field $\vec{B}$ is aligned with the $z$ axis. The electromagnetic field is assumed to be externally generated (and thus has no associated field equation), and only frozenfield configurations will be considered. The nucleon-meson couplings are denoted by $g$ and the electromagnetic couplings by $q$. The parameters of the model are the nucleon mass $m$, the masses of mesons $m_{\sigma}, m_{\omega}$, and $m_{\rho}$, and the nucleonmeson couplings. The self-interaction term with coupling constants $c_{\sigma}$ and $\lambda$ for the $\sigma$ meson are introduced. The RMF parametrization employed in this work is the FSUGold [52], where two more parameters $\xi$ and $\Lambda_{\omega}$ have been introduced: $\xi$ to describe the $\omega$ meson self-interactions, which soften the equation of state at high density, and $\Lambda_{\omega}$, a nonlinear mixed isoscalar-isovector term, which modifies the dependence of the symmetry energy. The FSUGold model has been chosen because it is frequently applied in the description of nuclear matter and stellar hadronic matter [53]. Although, FSUGold is too soft at large densities and it is not capable of describing a $2 M_{\odot}$ neutron star, we expect it will describe well nuclear matter below $3 \rho_{0}$, the range of densities we analyze.

From now we take the standard mean-field theory approach and display only some of the equations needed for this study. A complete set of equations and description of the method can be found in the literature (e.g., Refs. [47,51,54]). For the description of the system, we need the energy density of nuclear matter, the pressure, and the baryonic density. The energy density of nuclear matter can be expressed as

$$
\begin{aligned}
\varepsilon= & \varepsilon_{n}+\varepsilon_{p}+\frac{1}{2} m_{\sigma}^{2} \sigma^{2}+\frac{1}{3 !} c_{\sigma} g_{\sigma}^{3} \sigma^{3}+\frac{1}{4 !} \lambda g_{\sigma}^{4} \sigma^{4}+\frac{1}{2} m_{\omega}^{2} \omega_{0}^{2} \\
& +\frac{1}{8} \xi g_{\omega}^{4} \omega_{0}^{4}+\frac{1}{2} m_{\rho}^{2} \rho_{0}^{2}+3 \Lambda_{\omega} g_{\rho}^{2} \rho_{0}^{2} g_{\omega}^{2} \omega_{0}^{2},
\end{aligned}
$$

and the pressure of the system is obtained from the thermodynamical relation

$$
P_{m}=\sum_{i=n, p} \mu_{i} \rho_{i}-\varepsilon
$$

The proton and neutron chemical potentials read

$$
\begin{aligned}
& \mu_{p}=E_{F}^{p}+g_{\omega} \omega^{0}+\frac{1}{2} g_{\rho} \rho^{0}, \\
& \mu_{n}=E_{F}^{n}+g_{\omega} \omega^{0}-\frac{1}{2} g_{\rho} \rho^{0},
\end{aligned}
$$

where $E_{F}^{p}$ and $E_{F}^{n}$ are the proton and neutron Fermi energies related to their corresponding Fermi momenta $k_{F, v, s}^{p}$ and $k_{F, s}^{n}$ through

$$
\begin{gathered}
\left(k_{F, v, s}^{p}\right)^{2}=\left(E_{F}^{p}\right)^{2}-\left[\sqrt{m^{* 2}+2 v q_{p} B}-s \mu_{N} \kappa_{p} B\right]^{2}, \\
\left(k_{F, s}^{n}\right)^{2}=\left(E_{F}^{n}\right)^{2}-\bar{m}_{n s}^{2}
\end{gathered}
$$

where $v=n+\frac{1}{2}-\operatorname{sgn}(q) \frac{s}{2}=0,1,2, \ldots$ enumerates the Landau levels of the fermions with electric charge $q$, the quantum number $s$ is +1 for spin-up ( $\uparrow$ ) and -1 for spin-down
( $\downarrow$ ) particles, and for the neutrons we have introduced

$$
\bar{m}_{n s}=m^{*}-s \mu_{N} \kappa_{n} B,
$$

with $m^{*}$ the nucleon effective mass given by

$$
m^{*}=m-g_{\sigma} \sigma .
$$

The proton and neutron densities are given by

$$
\begin{gathered}
\rho_{p}=\frac{q_{p} B}{2 \pi^{2}} \sum_{\nu, s} k_{F, v, s}^{p}, \\
\rho_{n}=\frac{1}{2 \pi^{2}} \sum_{s}\left[\frac{1}{3}\left(k_{F, s}^{n}\right)^{3}-\frac{1}{2} s \mu_{N} \kappa_{n} B\right. \\
\left.\times\left(\bar{m}_{n s} k_{F, s}^{n}+\left(E_{F}^{n}\right)^{2}\left(\arcsin \left(\frac{\bar{m}_{n s}}{E_{F}^{n}}\right)-\frac{\pi}{2}\right)\right)\right],
\end{gathered}
$$

where the summation over the index $v$ in the expression for the proton density starts from 0 (1) for spin-up (spin-down) protons and runs up to the largest integer for which the square of the Fermi momentum of the proton is still positive. This maximum value of $v$ is defined by the ratio

$$
v_{\max }=\left[\frac{\left(E_{F}^{p}+s \mu_{N} \kappa_{p} B\right)^{2}-m^{* 2}}{2\left|q_{p}\right| B}\right] .
$$

Finally, the proton and neutron energy densities $\varepsilon_{p}$ and $\varepsilon_{n}$ that enter the total energy density (4) are given by

$$
\begin{aligned}
\varepsilon_{p}= & \frac{q_{p} B}{4 \pi^{2}} \sum_{\nu, s}\left[k_{F, v, s}^{p} E_{F}^{p}+\left(\sqrt{m^{* 2}+2 v q_{p} B}-s \mu_{N} \kappa_{p} B\right)^{2}\right. \\
& \left.\times \ln \left|\frac{k_{F, v, s}^{p}+E_{F}^{p}}{\sqrt{m^{* 2}+2 v q_{p} B}-s \mu_{N} \kappa_{p} B}\right|\right] \\
\varepsilon_{n}= & \frac{1}{4 \pi^{2}} \sum_{s}\left[\frac{1}{2} k_{F, s}^{n}\left(E_{F}^{n}\right)^{3}-\frac{2}{3} s \mu_{N} \kappa_{n} B\left(E_{F}^{n}\right)^{3}\right. \\
& \times\left(\arcsin \left(\frac{\bar{m}_{n s}}{E_{F}^{n}}\right)-\frac{\pi}{2}\right)-\left(\frac{1}{3} s \mu_{N} \kappa_{n} B+\frac{1}{4} \bar{m}_{n s}\right) \\
& \left.\left(\bar{m}_{n s} k_{F, s}^{n} E_{F}^{n}+\bar{m}_{n s}^{3} \ln \left|\frac{k_{F, s}^{n}+E_{F}^{n}}{\bar{m}_{n s}}\right|\right)\right] .
\end{aligned}
$$

\section{MAGNETIC SUSCEPTIBILITY}

The magnetization of nuclear matter defined as the derivative of the energy density with respect to $B$, at constant baryonic density $\rho$ and fixed proton fraction $Y_{p}$, can be written as

$$
\mathcal{M}=-\left.\frac{\partial \varepsilon}{\partial B}\right|_{\rho, Y_{p}}=\sum_{i=p, n}-\left.\frac{\partial \varepsilon_{i}}{\partial B}\right|_{\rho, Y_{p}}=\sum_{i=p, n} \mathcal{M}_{i}
$$

Note that since the density and the proton fraction is considered fixed, there is no contribution from the meson fields to the magnetization in this case. The proton magnetization, $\mathcal{M}_{p}$, is 
given by $[47,48,55]$

$$
\mathcal{M}_{p}=-\frac{\varepsilon_{p}}{B}+E_{F}^{p} \frac{\rho_{p}}{B}-\frac{q_{p} B}{2 \pi^{2}} \sum_{v, s} \bar{m}_{p v s} \ln \left|\frac{E_{F}^{p}+k_{F, v, s}^{p}}{\bar{m}_{p v s}}\right|\left(\frac{q_{p} v}{\tilde{m}_{p v s}}-s \mu_{N} \kappa_{p}\right),
$$

where $\bar{m}_{p v s}$ is defined as

$$
\bar{m}_{p v s}=\tilde{m}_{p v}-s \mu_{N} \kappa_{p} B
$$

with

$$
\tilde{m}_{p v}=\sqrt{m^{* 2}+2 q_{p} v B} .
$$

The magnetization of the neutrons $\mathcal{M}_{n}$ reads

$$
\begin{aligned}
\mathcal{M}_{n}= & \frac{1}{2 \pi^{2}} \sum_{s}\left(s \mu_{N} \kappa_{n}\right)\left\{\left(\frac{1}{6} \bar{m}_{n s}+\frac{1}{2} s \mu_{N} \kappa_{n} B\right) E_{F}^{n} k_{F, s}^{n}-\frac{1}{6}\left(E_{F}^{n}\right)^{3}\left(\arcsin \left(\frac{\bar{m}_{n s}}{E_{F}^{n}}\right)-\frac{\pi}{2}\right)\right. \\
& \left.-\bar{m}_{n s}^{2}\left(\frac{1}{2} s \mu_{N} \kappa_{n} B+\frac{1}{3} \bar{m}_{n s}\right) \ln \left|\frac{E_{F}^{n}+k_{F, s}^{n}}{\bar{m}_{n s}}\right|\right\} .
\end{aligned}
$$

The differential magnetic susceptibility of nuclear matter is calculated from the derivative of the magnetization with respect to the field $B$ for proton and neutron, at constant baryonic density

$$
\chi_{n}=\left.\frac{\partial \mathcal{M}_{n}}{\partial B}\right|_{\rho}
$$

For the proton we obtain the expression

$$
\begin{aligned}
\chi_{p}= & \frac{q_{p}}{2 \pi^{2}} \sum_{v, s}\left\{\frac{B E_{F}^{p}}{k_{F, v, s}^{p}}\left(\frac{q_{p} v}{\tilde{m}_{p v}}-s \mu_{N} \kappa_{p}\right)^{2}-2 \bar{m}_{p \nu s}\left(\frac{q_{p} v}{\tilde{m}_{p v}}-s \mu_{N} \kappa_{p}\right) \ln \left|\frac{E_{F}^{p}+k_{F, v, s}^{p}}{\bar{m}_{p v s}}\right|\right. \\
& \left.-B\left[\left(\frac{q_{p} v}{\tilde{m}_{p v}}-s \mu_{N} \kappa_{p}\right)^{2}-\bar{m}_{p v s} \frac{\left(q_{p} v\right)^{2}}{\tilde{m}_{p v}^{3}}\right] \ln \left|\frac{E_{F}^{p}+k_{F, v, s}^{p}}{\bar{m}_{p v s}}\right|\right\},
\end{aligned}
$$

whereas for the neutron we have

$$
\chi_{n}=\frac{1}{4 \pi^{2}} \sum_{s}\left(s \mu_{N} \kappa_{n}\right)^{2}\left\{E_{F}^{n} k_{F, s}^{n}+\bar{m}_{n s}\left(\bar{m}_{n s}+2 s \mu_{N} \kappa_{n} B\right) \ln \left|\frac{E_{F}^{n}+k_{F, s}^{n}}{\bar{m}_{n s}}\right|\right\} .
$$

At small values of the magnetic field $B$, we derive for the magnetization expressions similar to the ones derived in the nonrelativistic approach of Ref. [42]. We get for the proton spectrum

$$
E_{v s}^{p} \simeq \frac{k_{z v s}^{2}}{2 m^{*}}+m^{*}+\mu_{N} B\left[2 \frac{m}{m^{*}} n+\frac{m}{m^{*}}-s\left(\kappa_{p}+\frac{m}{m^{*}} \operatorname{sgn}(q)\right)\right],
$$

where $k_{z v s}$ is the component of the momentum parallel to the magnetic field. For the proton energy density we obtain in this limit

$$
\varepsilon_{p} \simeq \frac{q_{p} B}{2 \pi^{2}} \sum_{\nu, s}\left\{\frac{k_{F, v, s}^{p}{ }^{3}}{6 m^{*}}+m^{*} k_{F, v, s}^{p}+\mu_{N} B\left[2 \frac{m}{m^{*}} \nu+\frac{m}{m^{*}}-s\left(\kappa_{p}+\frac{m}{m^{*}} \operatorname{sgn}\left(q_{p}\right)\right)\right] k_{F, \nu, s}^{p}\right\} .
$$

Therefore, the expression the proton magnetization reads

$$
\begin{aligned}
\mathcal{M}_{p} & =-\left.\frac{\partial \varepsilon_{p}}{\partial B}\right|_{\rho, Y_{p}} \simeq-\frac{\varepsilon_{p}}{B}-\frac{q_{p} B}{2 \pi^{2}} \sum_{\nu, s}\left\{\mu_{N}\left[2 \frac{m}{m^{*}} \nu+\frac{m}{m^{*}}-s\left(\kappa_{p}+\frac{m}{m^{*}} \operatorname{sgn}\left(q_{p}\right)\right)\right] k_{F, v, s}^{p}\right\} \\
& \simeq-2 \frac{q_{p} B}{2 \pi^{2}} \sum_{\nu, s}\left\{\mu_{N}\left[2 \frac{m}{m^{*}} \nu+\frac{m}{m^{*}}-s\left(\kappa_{p}+\frac{m}{m^{*}} \operatorname{sgn}\left(q_{p}\right)\right)\right] k_{F, v, s}^{p}\right\}-\frac{q_{p}}{2 \pi^{2}} \sum_{\nu, s}\left\{\frac{k_{F, v, s}^{p}}{6 m^{*}}+m^{*} k_{F, v, s}^{p}\right\},
\end{aligned}
$$

from which, finally, we obtain the following approximated expression

$$
\mathcal{M}_{p}=2 \mu_{N}\left[\bar{\kappa}_{p} \mathrm{~W}_{p}-2 L-n_{p}\right]
$$

where the quantities $\bar{\kappa}_{p}, W_{p}, L$, and $n_{p}$ are defined as

$$
\bar{\kappa}_{p}=\kappa_{p}+\frac{m}{m^{*}}, \quad W_{p}=\frac{q_{p} B}{2 \pi^{2}} \sum_{v, s} s k_{F, v, s}^{p}
$$




$$
L=\frac{q_{p} B}{2 \pi^{2}} \sum_{\nu, s} \frac{m}{m^{*}} n k_{F, v, s}^{p}, \quad n_{p}=\frac{q_{p} B}{2 \pi^{2}} \sum_{\nu, s} \frac{m}{m^{*}} k_{F, v, s}^{p} .
$$

For the neutron magnetization we proceed in a similar way. Some details are given in the following. The interested reader is referred to Ref. [43] for a complete derivation. In the same fashion at small $B$, we get for the neutron spectrum

$$
E_{s}^{n} \simeq m^{*}+\frac{k_{s}^{2}}{2 m^{*}}-s \mu_{N} \kappa_{n} B=m^{*}+\frac{k_{z, s}^{2}+k_{\perp, s}^{2}}{2 m^{*}}-s \mu_{N} \kappa_{n} B,
$$

where $k_{z, s}$ and $k_{\perp, s}$ are the components parallel and orthogonal to the magnetic field. For the neutron energy density we obtain

$$
\varepsilon_{n} \simeq\left\{m^{*} \rho_{n}-\mu_{N} \kappa_{n} B W_{n}+\frac{1}{(2 \pi)^{2}} \sum_{s} \int \frac{k_{s}^{4}}{m^{*}} d k_{s}\right\},
$$

with

$$
W_{n}=\frac{1}{2 \pi^{2}} \sum_{s} \int s k_{s}^{2} d k_{s}
$$

being the spin asymmetry density for the neutron. The expression

$$
\mathcal{M}_{n}=-\left.\frac{\partial \varepsilon_{n}}{\partial B}\right|_{\rho, Y_{p}} \simeq \mu_{N} \kappa_{n} W_{n}
$$

is obtained for the neutron magnetization. We notice that this approximated expression was used for $\beta$-equilibrated stellar matter in Ref. [48].

\section{RESULTS AND DISCUSSION}

In the following we present and discuss the results obtained for nuclear matter under a strong magnetic field within the FSUGold parametrization of the NLW model [52]. We extend our analysis to baryon densities up to three times saturation density and magnetic field intensities within the range $10^{15} \mathrm{G} \leq B \leq 10^{19} \mathrm{G}$ in order to identify the sensitiveness of the strength of the magnetic field. As referred in the introduction, we consider in the following fields $B \leqslant 10^{19} \mathrm{G}$. Although there is no evidence that fields as high as $10^{19} \mathrm{G}$ exist in the crust, we include these values in the figures for low-density cases for the sake of completeness.

Spin-polarized isospin asymmetric nuclear matter can be seen as an infinite nuclear system composed by protons and neutrons. Each particle species, $i=n, p$, has two different fermionic components: spin-up particles $(\uparrow)$ and spin-down particles $(\downarrow)$, having number densities $\rho_{i}^{\uparrow}$ and $\rho_{i}^{\downarrow}$, respectively. The degree of spin polarization of the system can be studied through the relative polarization of the particle species $i$, defined by

$$
\Delta_{i}=\frac{\rho_{i}^{\uparrow}-\rho_{i}^{\downarrow}}{\rho_{i}^{\uparrow}+\rho_{i}^{\downarrow}} .
$$

Note that for small values of the magnetic field, the relation $\Delta_{i}=\rho_{i} W_{i}$ is fulfilled, with the quantities $W_{p}$ and $W_{n}$ defined previously. Note also that the value $\Delta_{n}=\Delta_{p}=0$ corresponds to nonpolarized (i.e., $\rho_{n}^{\uparrow}=\rho_{n}^{\downarrow}$ and $\rho_{p}^{\uparrow}=\rho_{p}^{\downarrow}$ ) matter, whereas $\Delta_{n}= \pm 1\left(\Delta_{p}= \pm 1\right)$ means that neutrons (protons) are totally polarized, i.e., all the neutron (proton) spins are align along the same direction.

In Fig. 1 we show the neutron relative polarization $\Delta_{n}$ in terms of the neutron density (left panel), and the proton
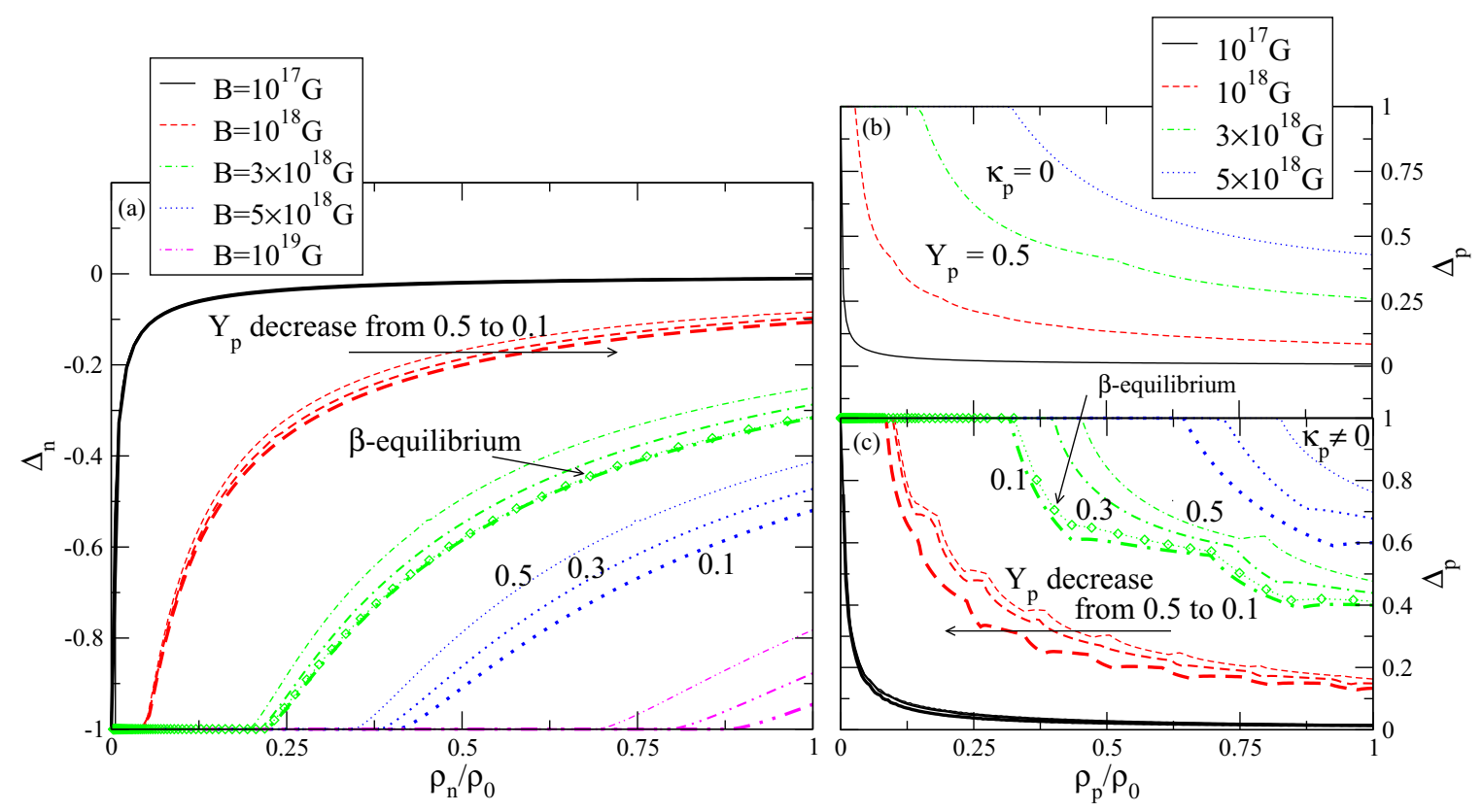

FIG. 1. (Color online) Neutron (left) and proton (right) relative polarizations as functions of their respective densities, for several values of magnetic field and for $Y_{p}=0.1,0.3,0.5$ and for $\beta$-equilibrium matter. For protons, the polarizations not including (top) or including (bottom) the anomalous magnetic moment are shown. 
relative polarization $\Delta_{p}$ as function of the proton density (right panel). Results for both different magnetic field intensities from $B=10^{17} \mathrm{G}$ to $B=5 \times 10^{18} \mathrm{G}$, and different proton fraction, $Y_{p}=0.1,0.3,0.5$, are plotted. Decreasing proton fractions are depicted with increasing line width. It should be pointed out that because only the neutron or the proton densities are shown, changing the proton fraction changes also the total density: A given neutron density for neutron-rich matter is attained at lower total densities than the same neutron density for symmetric matter.

We first discuss the results shown in the left panel of Fig. 1 for the neutron polarization. In this range of fields, for very low densities, neutrons are totally polarized (i.e., $\Delta_{n}=-1$ ), up to a critical density above which they become partially polarized. This is in agreement with other calculations of pure neutron matter (see, i.e., Refs. $[41,42,44,45]$ ). Neutrons have always a negative polarization due to the different sign of their coupling to the electromagnetic field with respect to that of the protons. By increasing the value of the magnetic field at a fixed proton fraction the critical neutron density increases. It is also seen that the critical neutron density is larger for the more asymmetric and neutron-rich matter, corresponding to a smaller total nucleon density. The same is true for polarized proton matter, see Fig. 1 right panel bottom: The total polarization occurs more easily in less dense matter, because the nucleon chemical potentials are smaller.

The critical neutron density depends on the proton fraction because changing $Y_{p}$ is equivalent to changing the neutron fraction $\left(Y_{n}=1-Y_{p}\right)$, and, for a given neutron density, as the total baryonic density increases, the more easily are neutrons totally polarized.

For a magnetic field of the order of $10^{17} \mathrm{G}$ neutrons are totally polarized only at very small densities, and for neutron densities above $0.02 \rho_{0}$ the partial polarization of neutrons is below $10 \%$. This low degree of polarization is due to its weak anomalous magnetic moment.

In Fig. 1(b) the relative proton polarization is plotted for symmetric matter without AMM. Once more, for very low densities, protons are totally polarized with $\Delta_{p}=1$, up to a critical density, where they become partially polarized with predominance of spin-up states, i.e., $0<\Delta_{p}<1$. The critical density increases with $B$. In Fig. 1(c) the AMM is included. The overall behavior does not change. When decreasing the proton fraction from 0.5 to 0.1 , the critical proton density decreases, associated with the increase of the total density. We have included in Figs. 1(a) and 1(c), respectively, the neutron and proton polarizations for $\beta$-equilibrium matter in the presence of a magnetic field $B=3 \times 10^{18} \mathrm{G}$. For neutrons the $\beta$-equilibrium polarization is practically coincident with the $Y_{p}=0.1$ results, and for protons, results for $Y_{p}=0.1$ are also quite similar, in agreement with proton fraction expected for $B=3 \times 10^{18} \mathrm{G}$ as shown in Fig. 2 where the proton fraction of $\beta$-equilibrium matter for different magnetic field intensities is shown for $\rho<4 \rho_{0}$.

In Fig. 3 we make a more careful analysis of the proton critical density as function of the magnetic field, with and without AMM and for two values of the proton fraction $Y_{p}=0.1$ and 0.5 . Protons are totally polarized on the region

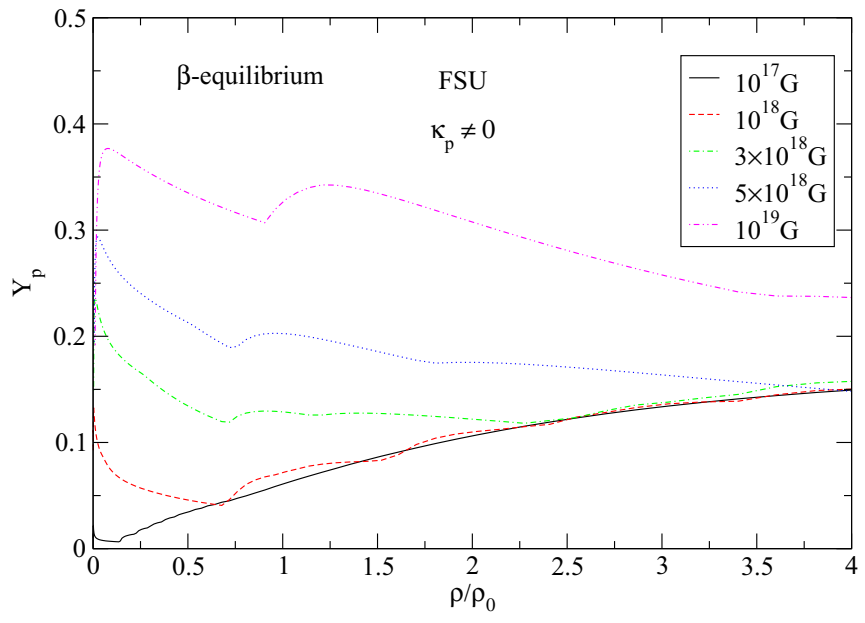

FIG. 2. (Color online) The proton fraction of $\beta$-equilibrium matter for several intensities of the magnetic field, taking into account the AMM.

below the lines of critical proton density. If no AMM is included, total polarization occurs when $e B>\frac{k_{F}^{p 2}}{2}$; however, with AMM the following conditions should be satisfied: $k_{F}^{p 2}<\left|4 \mu_{N} \kappa_{n} B\right| \sqrt{m^{* 2}+2 e B}$ for stronger fields or $k_{F}^{p 2}<$ $\left|2 \mu_{N} \kappa_{n} B\right|\left(\sqrt{m^{* 2}+2 e B}+\sqrt{m^{* 2}+4 e B}\right)-2 e B$ for weaker fields. The AMM favors the polarization so that the critical density is larger when the AMM is taken into account. For fields of the order of $10^{16} \mathrm{G}$ this difference is almost one order of magnitude larger, while at $B=10^{19} \mathrm{G}$ the difference reduces to a factor of two. We conclude, therefore, that a realistic calculation must include the AMM. The effect of the isospin asymmetry on the critical proton density is related with the filling of the Landau levels, which as referred before, depends on the total baryonic density.

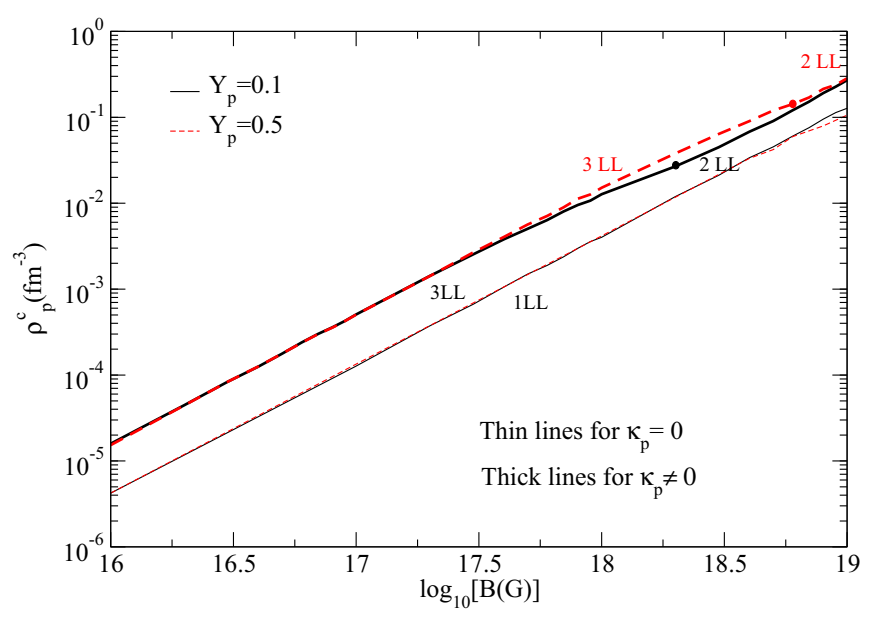

FIG. 3. (Color online) The critical density of protons as a function of the magnetic field, for $Y_{p}=0.1$ and 0.5 , and without or with AMM (thin or thick lines). The large dots indicate the filling of the indicated Landau level (LL). 

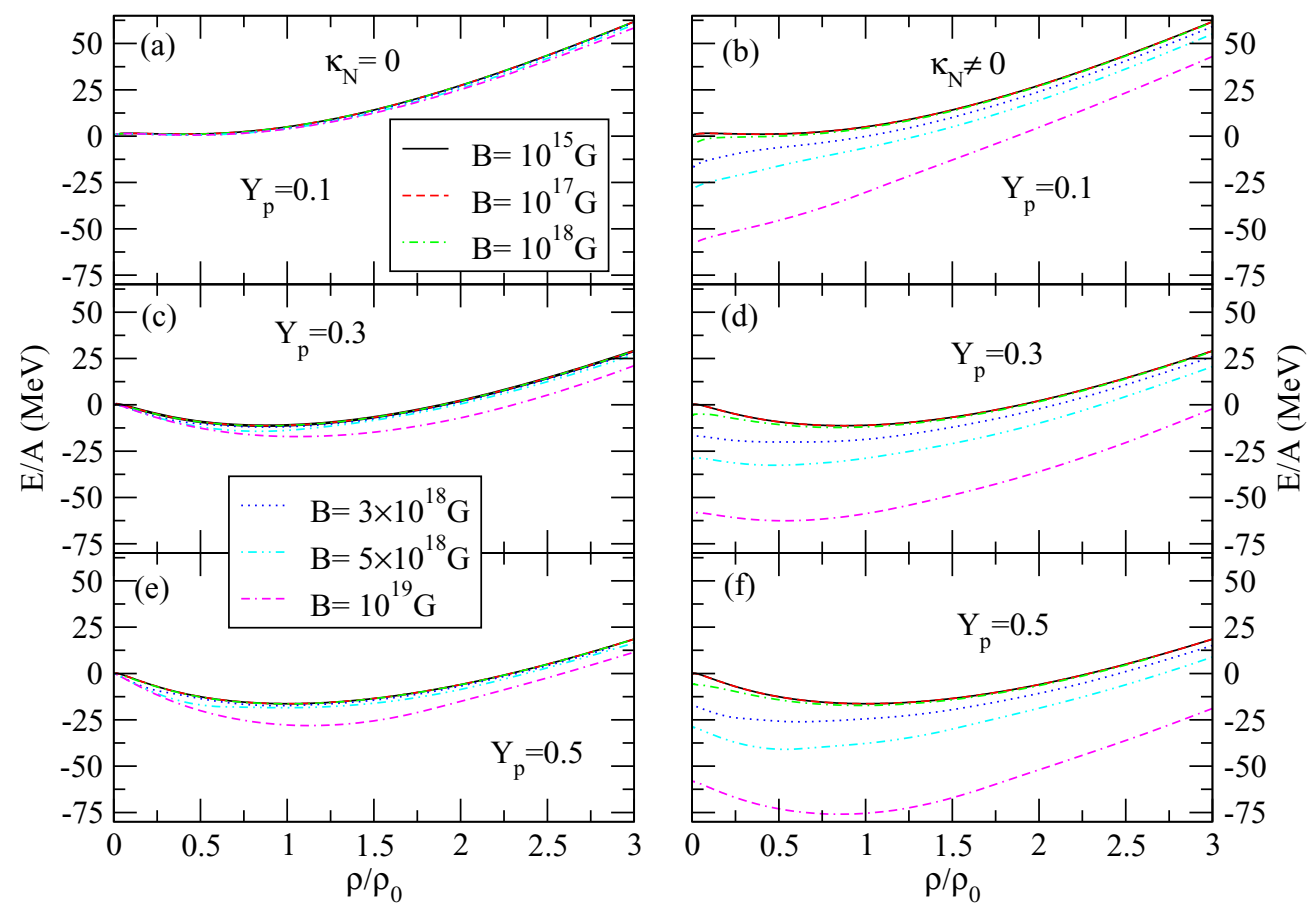

FIG. 4. (Color online) The energy per particle as a function of the density for several values of the magnetic field and proton fractions. In the left panel no AMM was included.

In Fig. 4 we show the energy per particle, defined by $E / A=$ $\frac{\varepsilon}{\rho}-m$, as a function of the baryon density, for several values of the magnetic field, and for $Y_{p}=0.1,0.3$, and 0.5. The curves obtained for $B<10^{18} \mathrm{G}$ are almost coincident. In Ref. [42], results obtained with $10^{14}<B<10^{18} \mathrm{G}$ also practically coincide; however, they are lower than the prediction for $B=0$. This could be due to some difference in the parametrization or normalization in the calculation done with $B=0$.

Ignoring the AMM (left panels), it is seen that in average the effect of the magnetic field is to increase the binding of the nuclear matter, and the effect is stronger for more symmetric matter. The AMM has a strong effect for larger values of the magnetic field. Note that for $B \geq 10^{18} \mathrm{G}$ there are already noticeable effects at densities $\rho \leq 0.25 \rho_{0}$. For stronger magnetic fields, the effect of the AMM leads clearly to an increase of the binding energy per particle.

Several authors have studied neutron matter under the effect of strong magnetic fields within different frameworks and interactions, such as the Gogny interaction $[31,32]$. Recently, the authors of Ref. [45] have used both microscopic, namely the Brueckner-Hartree-Fock approach with the Argonne V18 nucleon-nucleon potential supplemented with a three-body force, and phenomenological approaches, in particular an effective Skyrme model in a Hartree-Fock description and a mean-field quantum hadrodynamical formulation with the FSUGold parametrization.

In the following we present neutron matter properties and discuss how they change with the intensity of the magnetic field. In order to discuss the global state of polarization and bulk thermodynamical properties of pure neutron matter, we show in Fig. 5 (i) the neutron critical density corresponding to the transition from totally polarized as well as lines of partial polarization $(50 \%$ and $10 \%)$ as a function of the magnetic field [Fig. 5(a)], (ii) the energy per particle [Fig. 5(b)], (iii) the nucleonic pressure $P$ [Fig. 5(c)], and (iv) the compressibility $K$ [Fig. 5(d)], with the last three quantities as functions of the total density for several magnetic field intensities.

Just as we have discussed for protons, the neutron critical density is an increasing function of the magnetic field and the critical density is defined as the single-particle energy of neutrons is smaller than the energy required to start populating the neutron spin-up levels. This limit is defined by $\frac{k_{F}^{n 2}}{4 m^{*}}<$ $\left|\mu_{N} \kappa_{n} B\right|$. A magnetic field $B=3 \times 10^{16} \mathrm{G}$ has already a noticeable effect: A polarization of $10 \%$ is expected at $\rho_{n}=$ $0.001 \mathrm{fm}^{-3}$ and $50 \%$ for $\rho_{n}=0.0001 \mathrm{fm}^{-3}$. These are neutron densities of the order of the ones existing in the background neutron gas in the pasta phases of the inner crust.

From Fig. 5(b), it is seen that the effects due to magnetic fields start to be significant only for $B \geq 10^{18} \mathrm{G}$ at low baryon densities. For $B \leq 10^{17} \mathrm{G}$ neutron matter is not bound, as expected, because the magnetic field is too weak to have any effect on it. However, the binding increases when the intensity of the magnetic field grows, and for $B=10^{19} \mathrm{G}$ pure neutron matter is bound up to $\sim 1.5$ times saturation density. These results agree with the ones in Ref. [45].

The pressure in pure neutron matter is shown as a function of the baryonic density for several values of magnetic field in Fig. 5(c). Only the region corresponding to densities below the saturation density is plotted to show clearly the transition from totally polarized to partially polarized neutron matter. The pressure increases monotonically, showing, however, a softening at the onset of partially polarized matter. This 

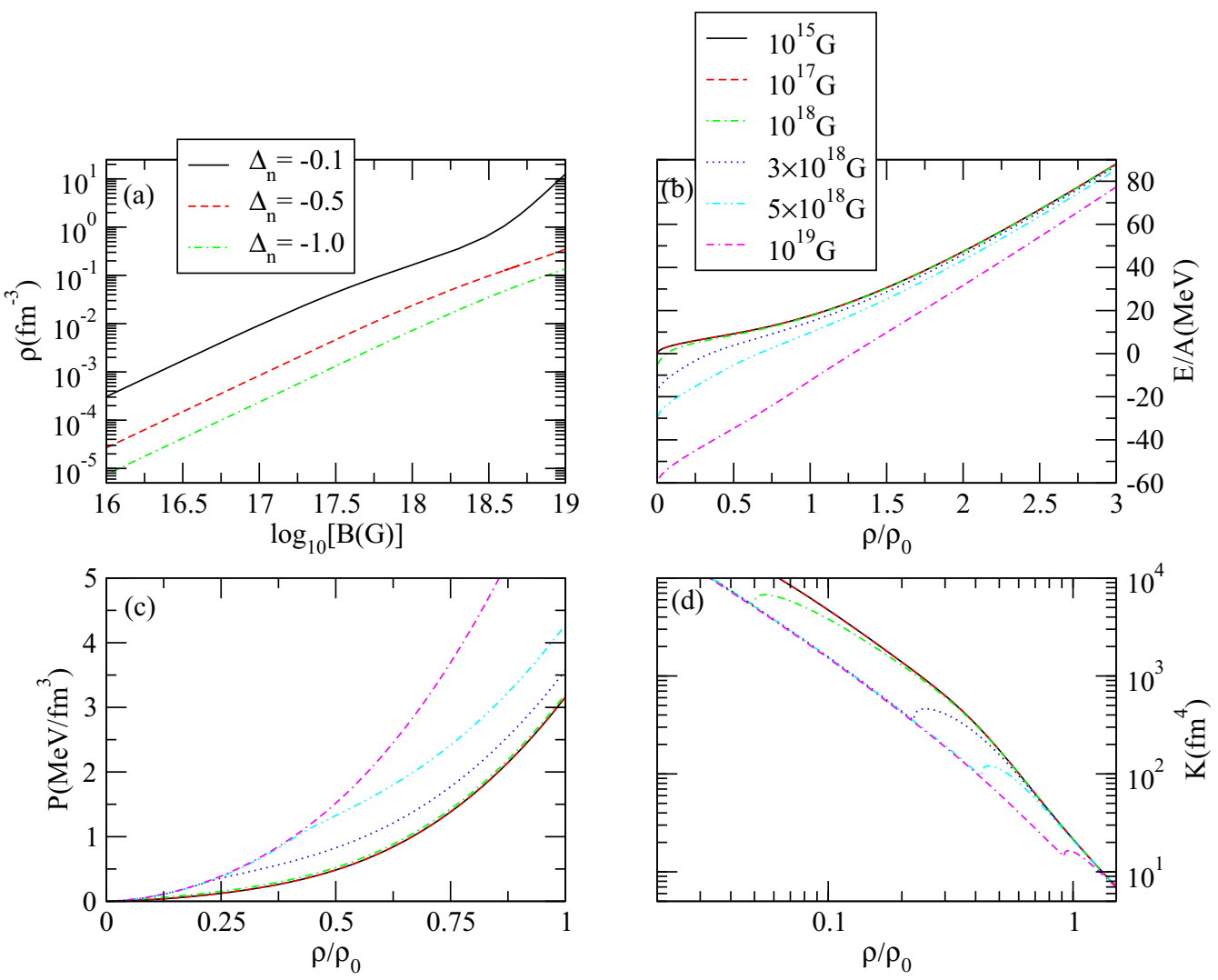

FIG. 5. (Color online) Several thermodynamic properties of neutron matter under the effect of an external magnetic field are shown: (a) the neutron critical density for total polarization and for partial polarization (50\% and 10\%) as a function of the magnetic field intensity, (b) the energy per particle, (c) the pressure, and (d) the compressibility $K$ are plotted as functions of the total neutron density for several magnetic field intensities. Matter below the full, dashed, and dot-dashed lines in panel (a) have, respectively, $\Delta_{n}<-0.1,-0.5$ and $\Delta_{n}=-1$.

transition is clearly seen on the isothermal compressibility $K$, defined through the first derivative of the pressure, i.e., $1 / K=\rho \frac{\partial P}{\partial \rho}$ [see Fig. 5(d)]. For each value of $B, K$ presents a kink at the critical density.

We next focus on the neutron magnetic susceptibility of asymmetric nuclear matter. In the following we consider both the magnetic susceptibility defined by the ratio $M_{n} / B$ and the differential susceptibility $\chi_{n}$. The dependence of the neutron magnetic and differential susceptibility on the magnetic field intensity is shown on, respectively, the left and right panels of Fig. 6. Results are shown for different total densities and proton fractions. As already found in Ref. [45] the magnitude of the neutron susceptibility is very small, $\chi_{n}<0.0015$ for FSU. Two different regimes are identified. In the low-field region corresponding to partially polarized matter, $B \leq 3 \times 10^{18} \mathrm{G}$, $M_{n} / B$ and $\chi_{n}$ exhibit a plateau. Beyond a threshold magnetic field, $M_{n} / B$ decreases, showing a change of the slope, clearly seen as the kink in $\chi_{n}$ which occurs at the transition from totally polarized to partially polarized matter. Above this critical magnetic field there is a strong decrease of the susceptibility.

The neutron susceptibility decreases if the neutron fraction decreases and has a nonmonotonic behavior with the density, increasing until $\sim \rho_{0}$ and decreasing above this density for the lower magnetic field intensities. This is clearly seen in
Fig. 7 where we show the magnetic $M_{n} / B$ [Fig. 7(a)] and the differential $\chi_{n}$ [Fig. 7(b)] neutron susceptibility as function of the baryon density, for several values of the magnetic field, and for a fixed proton fraction $Y_{p}=0.5$. At low densities $M_{n} / B$ practically does not change for $B<10^{18} \mathrm{G}$. For larger densities this range increases to fields one order of magnitude larger. $M_{n} / B$ increases linearly with $\rho$ at low densities when neutrons are totally polarized. At densities above the transition from totally polarized to partially polarized neutron matter, it continues increasing until a plateau is reached at high densities, which correspond to the limit when the terms with the magnetic field $B$ are negligible, $\chi_{n} \rightarrow \frac{1}{4 \pi^{2}}\left(\mu_{N} \kappa_{n}\right)^{2}\left[E_{F}^{n} k_{F}^{n}+\right.$ $\left.m^{* 2} \ln \left|\frac{E_{F}^{n}+k_{F}^{n}}{m^{*}}\right|\right]$. However, this value not always corresponds to the maximum of the magnetization for weak fields. For fields below $10^{18} \mathrm{G}$ the maximum occurs at $\sim 0.5-1.0 \rho_{0}$ before the plateau is attained.

The change of slope seen in $M_{n} / B$ corresponds to the kinks shown in the differential susceptibility $\chi_{n}$. We also show the ratio $M_{n} / B$ in the nonrelativistic limit, and contrary to the relativistic result the susceptibility does not saturate but increases monotonically with the density. The authors of Ref. [48] have obtained this same increasing trend by applying precisely the nonrelativistic limit of the ratio $M_{n} / B$. A similar behavior was also obtained in Ref. [45] with the Skyrme interaction, but in this case it could be that this is due to the 

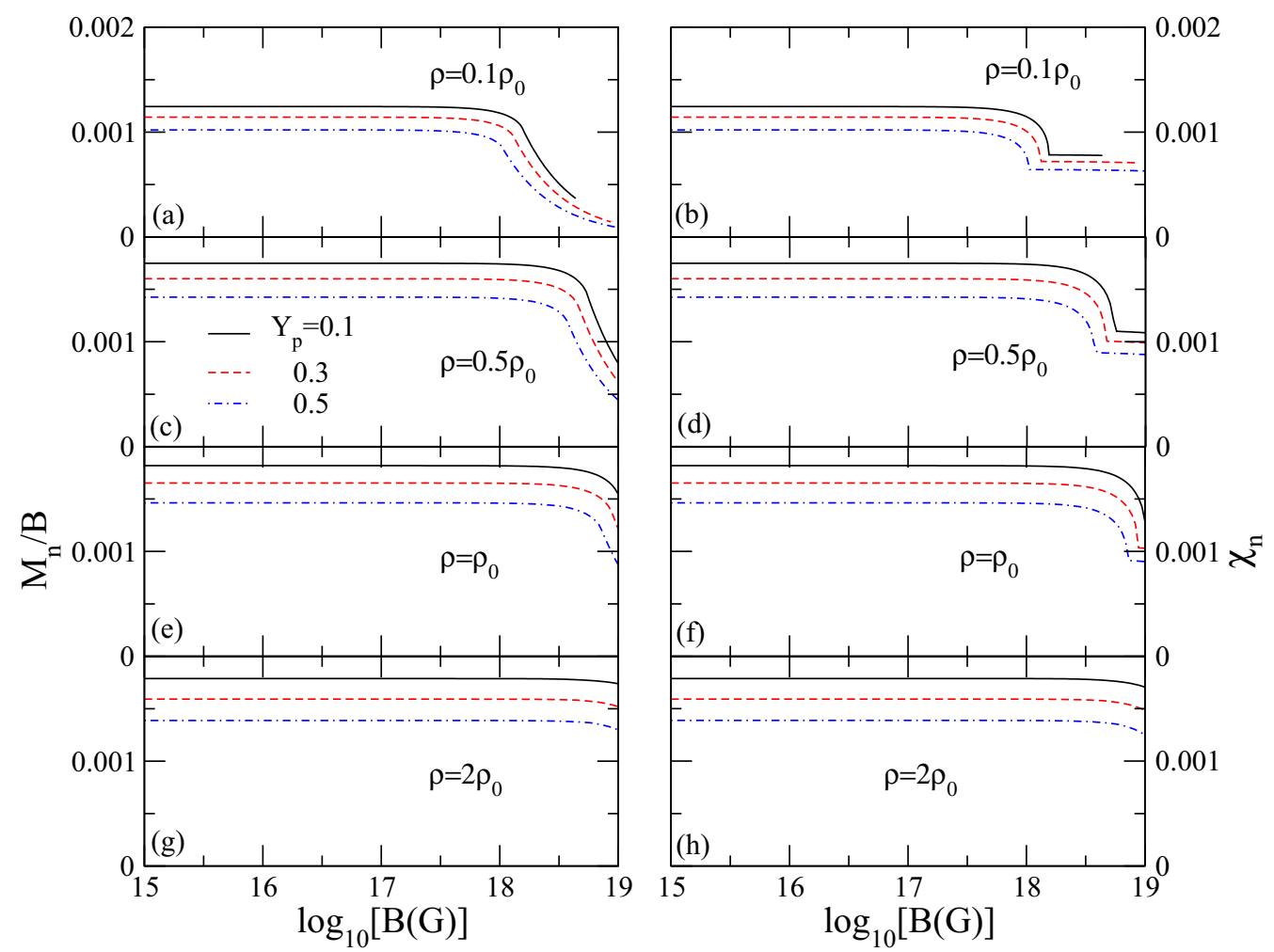

FIG. 6. (Color online) Neutron magnetic susceptibility (left panels) and differential susceptibility (right panels) as a function of the magnetic field and for several values of the density and isospin asymmetry.

properties of most Skyrme forces that predict a phase transition to a ferromagnetic phase at suprasaturation densities.

We now discuss the proton magnetization and compare it to the neutron one. The proton and neutron magnetic susceptibilities are presented in Fig. 8 as a function of the density for asymmetric nuclear matter under different intensities of the magnetic fields and for two proton fractions, $Y_{p}=0.1$ (left) and 0.3 (right). In the top and middle panels results for protons without (black solid line) and with (red [gray] dotted line) AMM, and for neutrons (green [gray] lines) are shown respectively for $B=10^{15} \mathrm{G}$ and $B=10^{17} \mathrm{G}$. In Figs. 8(e) and 8 (f) the different curves are for $B=10^{18} \mathrm{G}$ (black solid
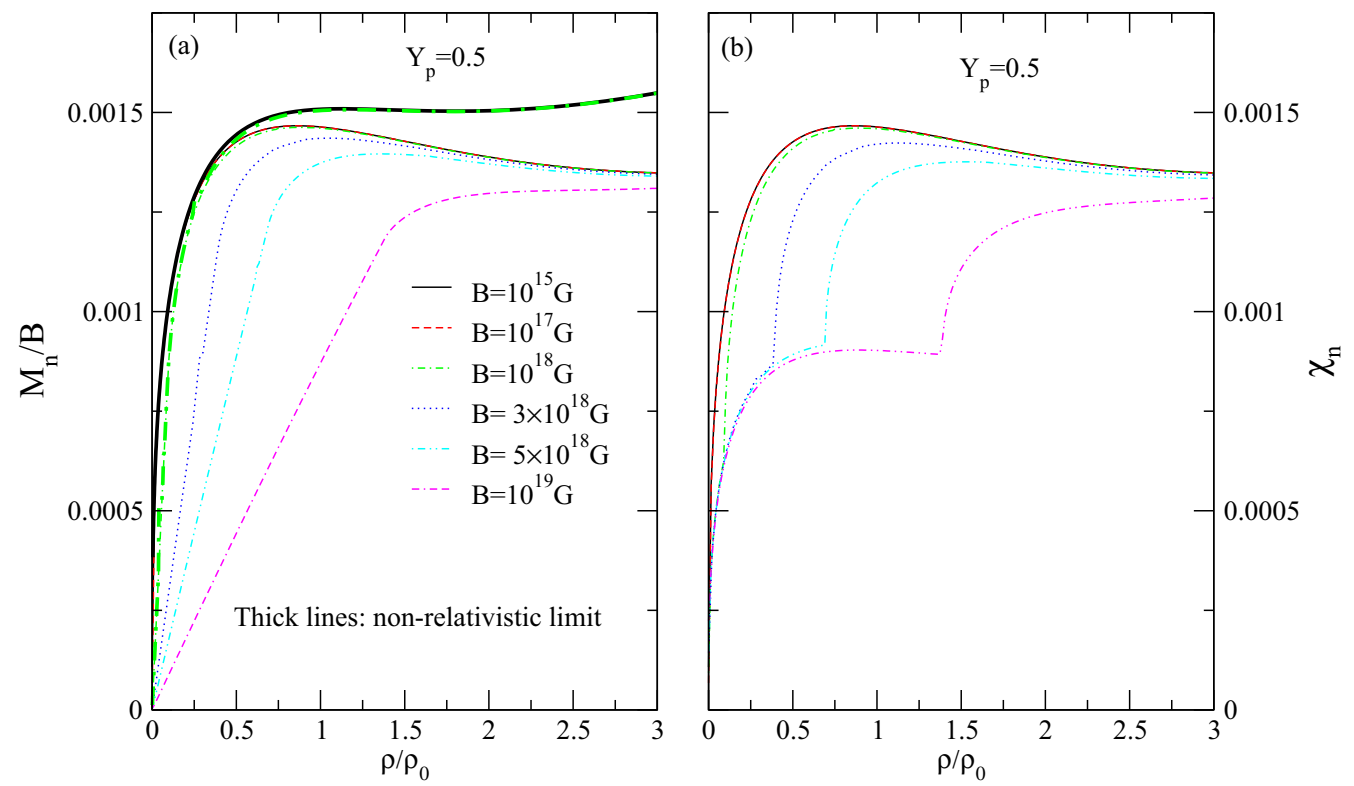

FIG. 7. (Color online) Neutron susceptibility (left) and differential susceptibility (right) as function of the baryon density, for several values of the magnetic field and for symmetric matter. The thick lines correspond to the nonrelativistic limit for $B=10^{15}$ and $10^{18} \mathrm{G}$. 

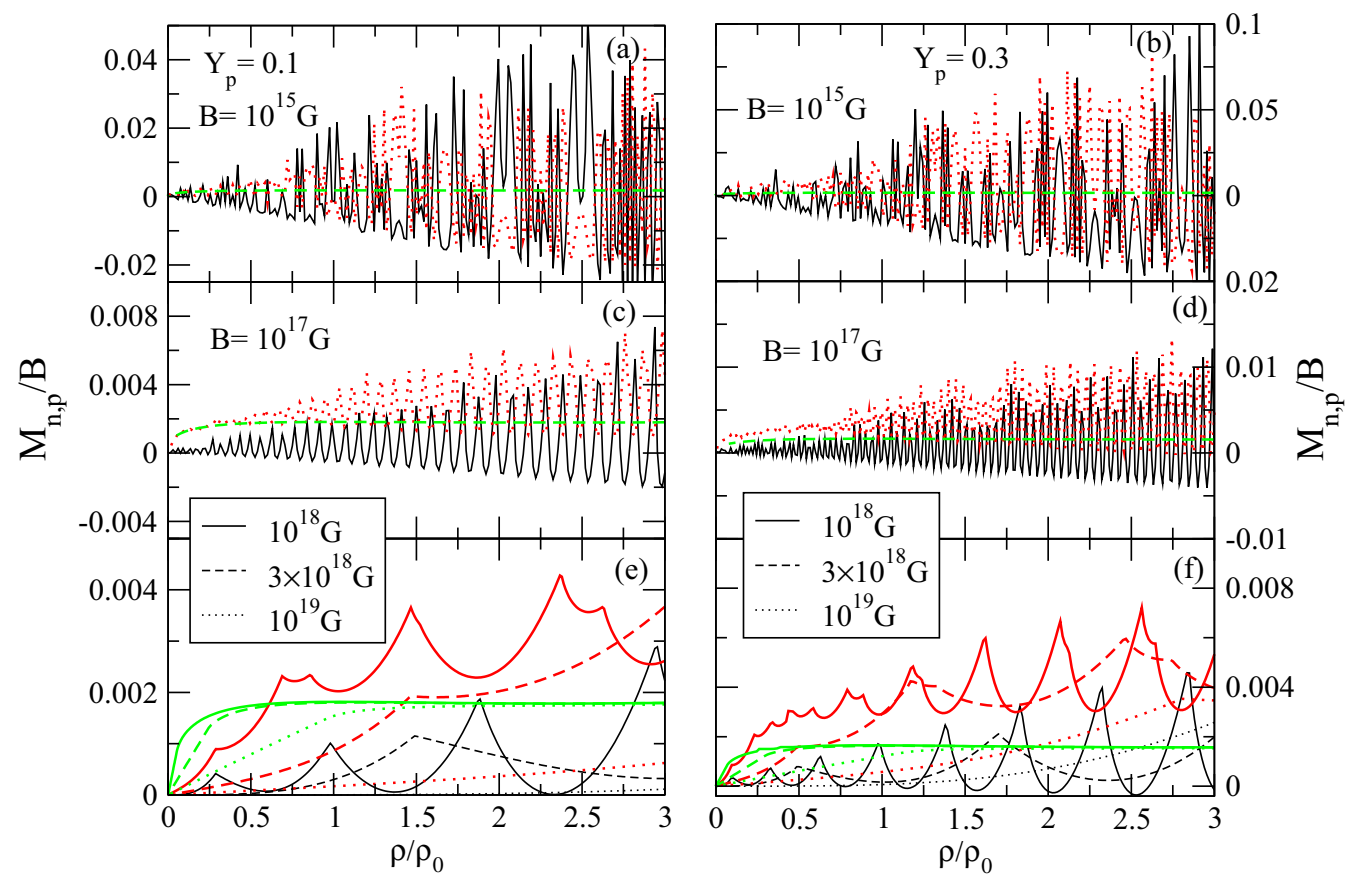

FIG. 8. (Color online) Proton and neutron magnetic susceptibilities as function of the density and for several values of the magnetic field. In the top and middle panels results with (without) AMM are represented by dotted (solid) lines for protons and dashed lines for neutrons. In the bottom panel results with (without) AMM for protons are given by the thick (thin) lines and the dashed lines are for neutrons.

line), $3 \times 10^{18} \mathrm{G}$ (red [gray] dashed line), and $10^{19} \mathrm{G}$ (blue [gray] dotted line), without and with AMM (thin and thick lines, respectively). In all the panels the neutron susceptibility is plotted with a green (gray) dashed curve. Please notice that the scale changes and the largest susceptibilities occur for the smallest magnetic fields.

The susceptibility curves for protons, at different magnetic fields, present the well-known de Haas van Alphen oscillations associated with the change in the number of Landau levels contributing at different fields. The filling of the levels becomes more complicated if the AMM is included and this is seen in the more complex structure of the magnetic susceptibility calculated with AMM; see bottom panel. Some of the main conclusions that may be drawn from these figures are (a) the proton magnetic susceptibility decreases much more strongly with $B$ than the neutron magnetic susceptibility, and if $B$ changes from $10^{15} \mathrm{G}$ to $10^{17} \mathrm{G}$ its magnitude changes by almost an order of magnitude, and the same if $B$ changes from $10^{17} \mathrm{G}$ to $10^{19} \mathrm{G}$, while the neutron susceptibility is practically unchanged; (b) the inclusion of the AMM may increase the proton susceptibility by a factor of two or more; (c) at low densities the proton and neutron magnetic susceptibilities are of the same order of magnitude and the fraction of protons defines how important is each contribution; (d) for $Y_{p}=0.1$ the neutron susceptibility is even larger than the proton one for subsaturation densities, such as the ones occurring in the crust of a neutron star.

In order to better understand the behavior at low densities we plot in Fig. 9 the magnetic susceptibilities for subsaturation densities and fields $B \leqslant 10^{17} \mathrm{G}$. These are field intensities that could exist in the inner crust of a magnetar. In fact, in the inner crust of a neutron star the background neutron gas will have densities that goes from zero to $\sim 0.5 \rho_{0}$. For neutron-rich matter with $Y_{p}=0.1$ and 0.3 or $\beta$-equilibrium matter the neutron susceptibilities are of the same order of magnitude. There is, however, a clear difference between the proton susceptibilities: As the proton fraction increases, the susceptibility becomes larger because the polarization is smaller. Total polarized matter has zero susceptibility. In particular, for these low fields $\beta$-equilibrium matter has a proton fraction below 0.1 (see Fig. 2), and, therefore, protons get more easily totally polarized.

This is matter that is totally or partially polarized as can be seen looking at Figs. 3 and 5: Pure neutron matter with $\rho_{n}=$ $0.0001 \mathrm{fm}^{-3}$ is totally polarized by a field $B \sim 6 \times 10^{16} \mathrm{G}$; for $\rho \sim 0.01 \mathrm{fm}^{-3}, Y_{p}=0.1$, and $B=10^{17} \mathrm{G}$ the proton polarization is almost $100 \%$ but the neutron polarization is one order of magnitude smaller. The magnetic susceptibility is larger for partially polarized matter and, although, at $\sim 0.5 \rho_{0}$ the maximum neutron magnetic susceptibility is attained for fields below $10^{18} \mathrm{G}$, at $\sim 0.02 \rho_{0}$ it has already a magnitude that is half of the maximum value. We expect that neutron polarization will affect the superfluidity of neutrons, reducing its fraction.

The effect of the proton fraction on the nucleon susceptibilities is also seen in Fig. 10 where these quantities are plotted as a function of the magnetic field intensity for several proton fractions and for $\rho=\rho_{0}$. Neglecting the AMM contribution makes the proton susceptibility go to zero as soon as only the first Landau level is occupied, and protons are totally polarized. This is expected taking the large $B$ limit of Eq. (23) with $\kappa_{p}=0$. For $Y_{p}=0.1$ the neutron susceptibility is of the order of the proton one or larger for $B>10^{17} \mathrm{G}$. Very strong oscillations occur for the weaker fields (or larger densities), 


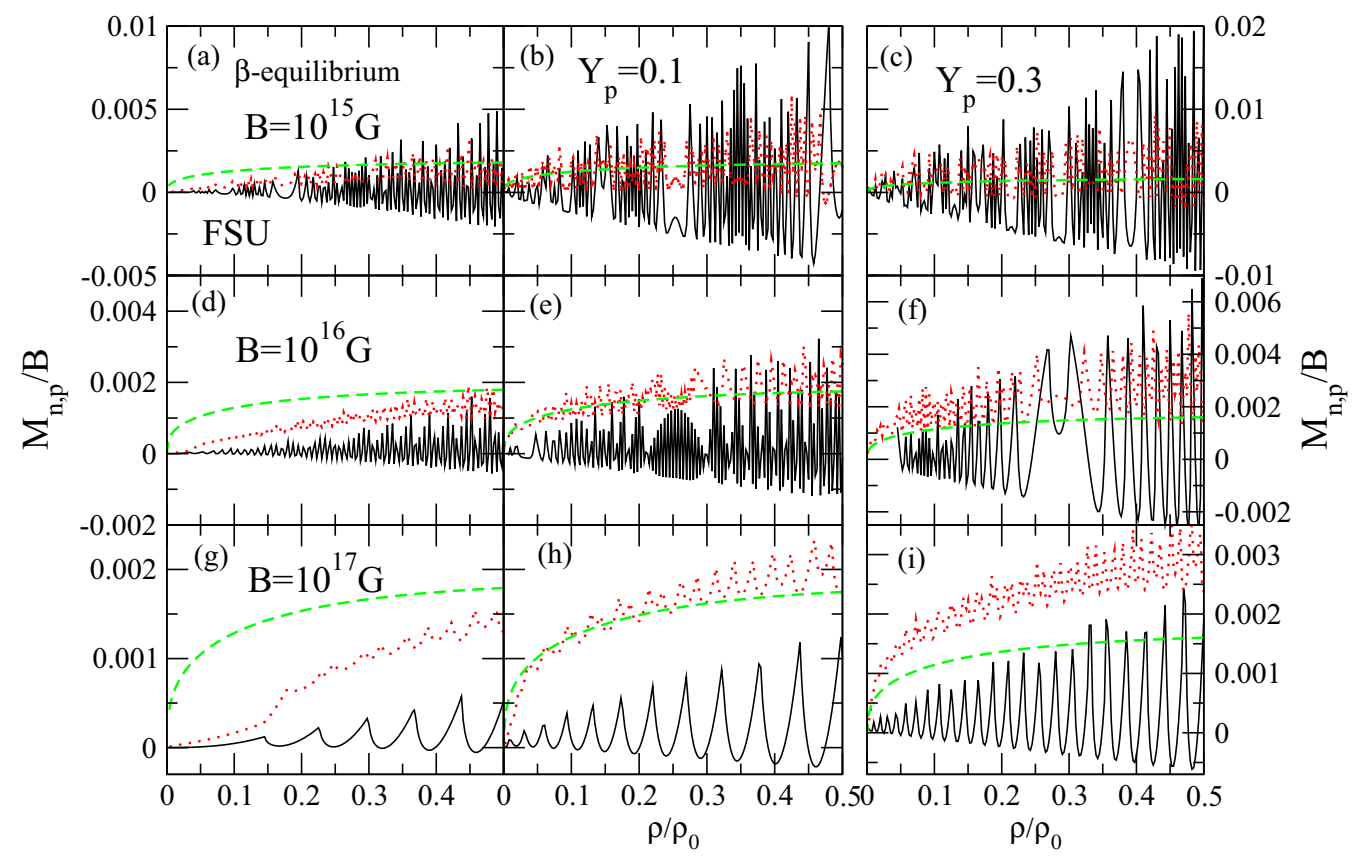

FIG. 9. (Color online) Proton and neutron magnetic susceptibilities as function of the density and for several values of the magnetic field, $B \leqslant 10^{17} \mathrm{G}$ and for densities of the interest for the neutron star crust. Results with (without) AMM are represented by dotted (solid) lines for protons and dashed lines for neutrons.

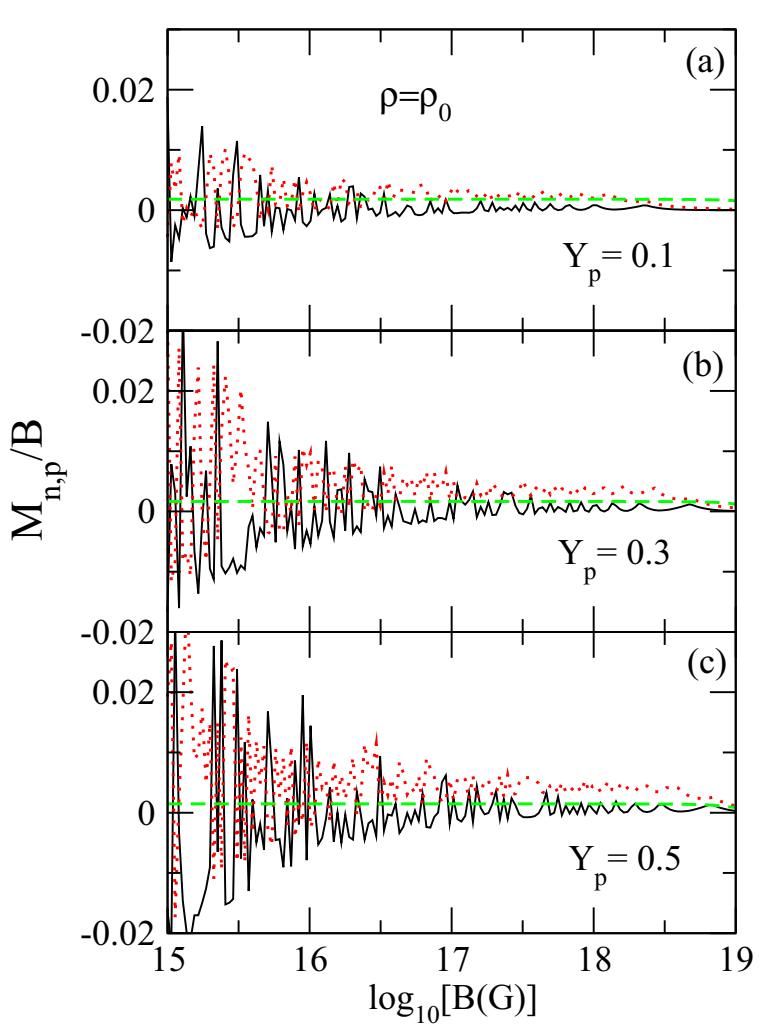

FIG. 10. (Color online) Proton and neutron magnetic susceptibilities and differential susceptibilities calculated at $\rho=\rho_{0}$ as function of the magnetic field for several values of the proton fraction, with and without AMM (dotted and solid lines, respectively) for protons and for neutrons (dashed line). and, therefore the differential susceptibilities have not been shown, but if an averaging is done as in Ref. [55] the average proton susceptibility would probably be of the order of the neutron one also for these fields (and densities). We also conclude that it is important to take into account AMM even for fields as small as $10^{16}-10^{17} \mathrm{G}$.

We have obtained an overall agreement with the conclusions obtained in previous works $[47,48]$, in particular, that the total magnetization decreases with an increasing magnetic field and that its magnitude is quite small.

The effect of the magnetic field on the proton fraction of $\beta$ equilibrium matter was discussed in Ref. [43]: At $\rho=0.1 \rho_{0} Y_{p}$ increases from $Y_{p}<0.005$ at $10^{15} \mathrm{G}$ to 0.18 at $3.6 \times 10^{18} \mathrm{G}$. However, this effect is much smaller at larger densities: for $\rho=2 \rho_{0}, 4 \rho_{0}, Y_{p}$ increases from $Y_{p}=0.15,0.22$ at $10^{15} \mathrm{G}$ to, respectively, $Y_{p}=0.16$ and 0.23 at $3.6 \times 10^{18} \mathrm{G}$. These results are in agreement with the proton fractions plotted in Fig. 2. For the indicated densities $\left(0.1 \rho_{0}, 2 \rho_{0}, 4 \rho_{0}\right)$, the total proton polarization is attained, respectively, at $\log _{10}[B(G)]=$ $16.2,18.4$, and 18.8. These results are confirmed in our present work as particular cases of a more systematic study; see Fig. 3. Similar conclusions with respect to the effect of the magnetic field on the proton fraction have been drawn in Ref. [51], where it is shown that, for $B=4.4 \times 10^{18} \mathrm{G}$, the magnetic field affects mainly densities below $2 \rho_{0}$ and, instead of the usual proton fractions below 0.1 , proton fractions above this value are expected. An increase of the proton fraction disfavors polarization, and, therefore, in $\beta$-equilibrium magnetized matter the proton total polarization will not occur as easily.

In matter with neutrino trapping it was shown in Ref. [50] that at low densities neutrino suppression occurs due to the 
larger proton fractions, which can be as high as 0.4. Larger amounts of protons mean that polarization effects of the magnetic field will be smaller on the protons and larger on neutrons. On the other hand, the contribution of protons (neutrons) to the total magnetization will increase (decrease).

At subsaturation densities matter is not homogeneous and a pasta phase calculation is required. In Ref. [49] a first study was performed and it was shown that fields below $10^{18} \mathrm{G}$ have a non-negligible effect on the pasta structure. In particular, it was shown that the magnetic field disfavors neutron drip and, therefore, the neutron gas outside the cluster is less dense for a given density and total polarization occurs more easily.

In stellar $\beta$-equilibrium neutral matter besides protons and neutrons also the contribution of electrons (and muons above $0.12 \mathrm{fm}^{-3}$ ) should be considered as discussed in Refs. [43,51]. Consequently a complete description of stellar matter requires also the leptonic contribution for the description of quantities such as the total magnetization; see Ref. [48].

\section{SUMMARY AND CONCLUSIONS}

In the present work we have studied the proton and neutron polarization and magnetic susceptibility of asymmetric nuclear matter within a relativistic mean-field approach, in particular, the FSUGold parametrization.

The calculations were performed at a fixed proton fraction, and for the proton results with and without AMM were compared. We have calculated independently the proton and the neutron magnetic susceptibilities and compared their magnitudes. Both of them are quite small, indicating that the magnetization induced by an external magnetic field is weak. Similar conclusions have been obtained in Refs. [47,48].

The proton susceptibility oscillates very strongly due to the filling of Landau levels and decreases with an increasing magnetic field. It was shown that at subsaturation densities the susceptibility calculated including the AMM may be several times larger than the results obtained when it is ignored, for magnetic fields with an intensity larger than $\sim 5 \times 10^{16} \mathrm{G}$, and, therefore, it is important to take into account AMM for fields in the range $10^{16}-10^{17} \mathrm{G}$.

The neutron susceptibility has a behavior very different not only because it does not oscillate since the neutron has zero electric charge but also because at large densities it converges to a value that is independent of the magnetic field while the proton susceptibility increases with the density for a fixed value of $B$. However, it was also shown that in the nonrelativistic limit neutron susceptibility increases monotonically with density. We have shown that at low density and for small proton fractions the neutron susceptibility may be as large as the proton one or even larger.

We have also calculated the transition density from partially to totally polarized matter as a function of the magnetic field intensity and it was shown that neutron matter is totally polarized by a field $6 \times 10^{16} \mathrm{G}$ and $\rho=0.0001 \mathrm{fm}^{-3}$. The same field will also totally polarize the protons of asymmetric nuclear matter at $\rho=0.002 \mathrm{fm}^{-3}$ with $Y_{p}=0.1$. This behavior occurs for densities of relevance in the neutron star crusts and we expect that neutron superfluidity and transport properties of the crust will be affected by the presence of magnetic fields at least as strong as $10^{16} \mathrm{G}$. This has been studied for the opacity, e.g., in Ref. [56]. In fact, at low densities, such as the ones occurring in the inner crust, it is expected neutron superfluidity in the attractive channel ${ }^{1} S_{0}$. A partial or total neutron polarization will naturally hinder the formation of neutron pairing. The consequences of the nonexistence or reduction of neutron superfluidity would be a faster cooling of low-mass neutron stars, stars for which the direct Urca processes are not expected, and a reduction of the glitch phenomena since the neutron pairing determines the vortex structure [57-59]. Also, a reduction of the susceptibility would have strong effects on the mean free path of a neutrino in dense matter, and, therefore, on the cooling of the star [24].

\section{ACKNOWLEDGMENTS}

One of the authors (A.R.) acknowledges J. da Providência for many helpful and elucidating discussions. This work is partly supported by the project PEst-OE/FIS/UI0405/2014 developed under the inititative QREN financed by the UE/FEDER through the program COMPETE-"Programa Operacional Factores de Competitividade" and by "NewCompstar," COST Action MP1304. M.A.P.G. would like to thank partial financial support from FIS2012-30926 MINECO and MULTIDARK projects.
[1] B. A. Li, A. Ramos, G. Verde, and I. Vidaña, eds., Nuclear Symmetry Energy, special issue of Eur. Phys. J A 50 (2014).

[2] V. Baran, M. Colonna, V. Greco, and M. Di Toro, Phys. Rep. 410, 335 (2005).

[3] B. A. Li B, W. Chen, and C. M. Ko, Phys. Rep. 464, 113 (2008).

[4] A. W. Steiner, M. Prakash, J. Lattimer, and P. J. Ellis, Phys. Rep. 411, 325 (2005).

[5] A. K. Harding and D. Lai, Rep. Prog. Phys. 69, 2631 (2006).

[6] T. Tatsumi, T. Maruyama, E. Nakano, and K. Nawa, Nucl. Phys. A 774, 827 (2006).

[7] C. Thompson and R. C. Duncan, Astrophys. J. 408, 194 (1993).

[8] D. H. Brownell and J. Callaway, Nuovo Cimento B 60, 169 (1969).

[9] M. J. Rice, Phys. Lett. A 29, 637 (1969).
[10] J. W. Clark and N. C. Chao, Lett. Nuovo Cimento 2, 185 (1969).

[11] J. W. Clark, Phys. Rev. Lett. 23, 1463 (1969).

[12] S. D. Silvertein, Phys. Rev. Lett. 23, 139 (1969).

[13] E. Østgaard, Nucl. Phys. A 154, 202 (1970).

[14] J. M. Pearson and G. Saunier, Phys. Rev. Lett. 24, 325 (1970)

[15] V. R. Pandharipande, V. K. Garde, and J. K. Srivastava, Phys. Lett. B 38, 485 (1972).

[16] S. O. Bäckman and C. G. Källman, Phys. Lett. B 43, 263 (1973).

[17] P. Haensel, Phys. Rev. C 11, 1822 (1975).

[18] A. D. Jackson, E. Krotscheck, D. E. Meltzer, and R. A. Smith, Nucl. Phys. A 386, 125 (1982).

[19] M. Kutschera and W. Wójcik, Phys. Lett. B 223, 11 (1989); 325, 271 (1994). 
[20] S. Marcos, R. Niembro, M. L. Quelle, and J. Navarro, Phys. Lett. B 271, 277 (1991); P. Bernardos, S. Marcos, R. Niembro, and M. L. Quelle, ibid. 356, 175 (1996).

[21] A. Vidaurre, J. Navarro, and J. Bernabeu, Astron. Astrophys. 135, 361 (1984).

[22] A. Rios, A. Polls, and I. Vidaña, Phys. Rev. C 71, 055802 (2005).

[23] D. López-Val, A. Rios, A. Polls, and I. Vidaña, Phys. Rev. C 74, 068801 (2006).

[24] S. Fantoni, A. Sarsa, and K. E. Schmidt, Phys. Rev. Lett. 87, 181101 (2001).

[25] I. Vidaña, A. Polls, and A. Ramos, Phys. Rev. C 65, 035804 (2002).

[26] I. Vidaña and I. Bombaci, Phys. Rev. C 66, 045801 (2002).

[27] I. Bombaci, A. Polls, A. Ramos, A. Rios, and I. Vidaña, Phys. Lett. B 632, 638 (2006).

[28] F. Sammarruca and P. G. Krastev, Phys. Rev. C 75, 034315 (2007).

[29] F. Sammarruca, Phys. Rev. C 83, 064304 (2011).

[30] M. Bigdeli, Phys. Rev. C 82, 054312 (2010).

[31] M. A. Pérez-García, J. Navarro, and A. Polls, Phys. Rev. C 80, 025802 (2009).

[32] M. A. Pérez-García, Phys. Rev. C 80, 045804 (2009).

[33] C. Y. Cardall, M. Prakash, and J. M. Lattimer, Astrophys. J. 554, 322 (2001).

[34] A. Broderick, M. Prakash, and J. M. Lattimer, Phys. Lett. B 531, 167 (2002).

[35] S. A. Olausen and V. M. Kaspi, Astrophys. J. Suppl. 212, 6 (2014).

[36] R. Turolla, S. Zane, J. A. Pons, P. Esposito, and N. Rea, ApJ 740, 105 (2011).

[37] J. Frieben and L. Rezzolla, MNRAS 427, 3406 (2012).

[38] D. Chatterjee, T. Elghozi, J. Novak, and M. Oertel, MNRAS 447, 3785 (2015).

[39] M. Ruderman, T. Zhu, and K. Chin, Astrophys. J. 482, 267 (1998).

[40] V. R. Khalilov, Phys. Rev. D 65, 056001 (2002).

[41] M. A. Pérez-García, Phys. Rev. C 77, 065806 (2008).

[42] R. Aguirre, Phys. Rev. C 83, 055804 (2011).

[43] M. A. Pérez-García, C. Providência, and A. Rabhi, Phys. Rev. C 84, 045803 (2011).
[44] R. Aguirre and E. Bauer, Phys. Lett. B 721, 136 (2013).

[45] R. Aguirre, E. Bauer, and I. Vidaña, Phys. Rev. C 89, 035809 (2014).

[46] R. D. Blandford and L. Hernquist, J. Phys. C: Solid State Phys. 15, 6233 (1982).

[47] A. Broderick, M. Prakash, and J. M. Lattimer, Astrophys. J. 537, 351 (2000).

[48] J. Dong, W. Zuo, and J. Gu, Phys. Rev. D 87, 103010 (2013).

[49] R. C. R. de Lima, S. S. Avancini, and C. Providência, Phys. Rev. C 88, 035804 (2013).

[50] A. Rabhi and C. Providência, J. Phys. G37, 075102 (2010)

[51] A. Rabhi, C. Providência, and J. da Providência, J. Phys. G: Nucl. Part. Phys. 35, 125201 (2008).

[52] B. G. Todd-Rutel and J. Piekarewicz, Phys. Rev. Lett. 95, 122501 (2005).

[53] J. Piekarewicz, F. J. Fattoyev, and C. J. Horowitz, Phys. Rev. C 90, 015803 (2014); F. J. Fattoyev and J. Piekarewicz, Phys. Rev. Lett. 111, 162501 (2013); M. Hempel, V. Dexheimer, S. Schramm, and I. Iosilevskiy, Phys. Rev. C 88, 014906 (2013); N. Gupta and P. Arumugam, ibid. 88, 015803 (2013); F. J. Fattoyev, J. Carvajal, W. G. Newton, and B. A. Li, ibid. 87, 015806 (2013); S. S. Avancini, C. C. Barros, L. Brito, S. Chiacchiera, D. P. Menezes, and C. Providência, ibid. 85, 035806 (2012); F. Grill, C. Providência, and S. S. Avancini, ibid. 85, 055808 (2012).

[54] S. Chakrabarty, Phys. Rev. D 54, 1306 (1996); S. Chakrabarty, D. Bandyopadhyay, and S. Pal, Phys. Rev. Lett. 78, 2898 (1997).

[55] X.-G. Huang, M. Huang, D. H. Rischke, and A. Sedrakian, Phys. Rev. D 81, 045015 (2010).

[56] M. Ángeles Pérez-García, Eur. Phys. J. A 44, 77 (2010).

[57] M. Baldo, E. E. Saperstein, and S. V. Tolokonnikov, Nucl. Phys. A 749, 42 (2005); D. J. Dean and M. Hjorth-Jensen, Rev. Mod. Phys. 75, 607 (2003).

[58] D. Page, J. M. Lattimer, M. Prakash, and A. W. Steiner, Astrophys. J. 707, 1131 (2009).

[59] B. Carter, in Lectures Notes in Physics Vol. 578, edited by D. Blaschke, N. K. Glendenning, and A. Sedrakian (Springer, Berlin, 2000), p. 54; A. Sedrakian, Phys. Rev. D 71, 083003 (2005). 